\title{
Diffuse cerebral vasospasm after resection of schwannoma: a case report
}

This article was published in the following Dove Press journal:

Neuropsychiatric Disease and Treatment

5 February 2015

Number of times this article has been viewed

Ji Qi

Liwei Zhang

Wang Jia

Junting Zhang

Zhen Wu

Department of Neurosurgery, Beijing Tiantan Hospital, Capital Medical University, Beijing, People's Republic of China
Correspondence: Liwei Zhang

Department of Neurosurgery,

Beijing Tiantan Hospital, Capital Medical

University, No. 6, Road Tiantanxili,

Beijing I00050, People's Republic of

China

Tel +86 I33 8I $2 \mid 8298$

Fax +86 2I 64085875

Email zlwtt@aliyun.com
Background: The etiology of vasospasm after brain tumor resection remains unclear. This is the first report of diffuse cerebral vasospasm following resection of a schwannoma located in the left portion/part of the ventral medulla oblongata.

Case presentation: A 16-year-old boy presented with dizziness and projectile vomiting without apparent cause three months before admission. Magnetic resonance imaging revealed a space-occupying lesion in the left portion of the ventral medulla oblongata. The tumor was completely resected. Pathological examination was consistent with diagnosis of schwannoma. Three days after surgery, the patient was in an unconscious state. Magnetic resonance angiography revealed the occurrence of cerebral vasospasm, with ischemic infarction and segmental cerebrovascular stenosis. Seven days after surgery, dehydration therapy was carried out and decompressive craniectomy was performed in the frontal and temporal areas. Despite treatments, the patient was in a persistent vegetative state after surgery, with normal vital signs.

Conclusion: This was an uncommon occurrence of diffuse cerebral vasospasm following the resection of schwannoma. Appropriate management of this condition is uncertain, but aggressive treatments including expansion of blood volume and relief of spasm should be performed to treat postsurgery vasospasm.

Keywords: vasospasm, cerebral tumor resection, schwannoma, decompressive craniectomy, magnetic resonance angiography

\section{Introduction}

Cerebral vasospasm is a complication usually seen after intracranial aneurysm rupture or traumatic brain injury. This condition may easily be confused with cerebral edema or surgical injury, leading to misdiagnosis and inappropriate treatments. ${ }^{1}$ A symptomatic vasospasm following brain tumor surgery is rare, and even rarer in children and adolescents. ${ }^{2,3}$

This is a case study of a patient with diffuse cerebral vasospasm following resection of schwannoma.

\section{Case report}

A 16-year-old Chinese boy presented with dizziness and projectile vomiting for 3 months. He was admitted at the Neurosurgery Department of Beijing Tiantan Hospital in November 2009 after a progressive exacerbation of symptoms, with unsteady walking and increased blood pressure. Physical examination revealed lucidity, equal reaction of pupils to light, full eye movement, facial symmetry, lolling and muscle dystrophy of the left part of the tongue, bilateral gag hyporeflexia, soft neck, grade V limbs muscle strength, normal muscle tone, Rombergism positive, and bilaterally positive Babinski’s sign. Blood pressure was 142/110 mmHg. 
Computed tomography (CT) revealed an intracranial space-occupying lesion. Magnetic resonance imaging (MRI) confirmed the lesion (size: $2.5 \times 2.0 \times 2.0 \mathrm{~cm}$ ) in the left part of the ventral medulla oblongata (Figure 1A). The lesion was significantly enhanced after contrast injection. The medulla oblongata was extruded, with peritumoral edema. The patient was a full-term delivery and had no family history of hypertension and coronary heart disease.

Tumor resection was performed in the right lateral position. Surgical time was 6 hours including 2.5 hours for tumor removal. The left vertebral artery and posterior cranial nerve were located on the surface of the tumor. The tumor was completely removed by resection under a microscope. After resection, the left vertebral artery and posterior cranial nerve remained intact. Total intraoperative bleeding was $300 \mathrm{~mL}$. Blood loss due to the tumor resection per se was about $50 \mathrm{~mL}$. The surrounding tissues were shielded using gauze and all blood leaking was suctioned, leaving almost no blood in the arachnoid space. Pathological examination was consistent with diagnosis of schwannoma. Indeed, hematoxylin and eosin staining (Figure 2) identified the classic Antoni A and Antoni B regions. In the Antoni A region, cells were closely arranged with a palisade-like structure. In the Antoni B region, the cells were arranged loosely. Immunohistochemical analysis was then performed, which revealed that the tumor was S100 positive, EMA negative, and GFAP negative, which was consistent with the schwannoma. ${ }^{4}$

At day 1 after surgery, the pupils were equally sensitive to light. Wound dressing was clean and dry. The patient presented airway obstruction, and tracheal intubation was performed to secure airway patency. The airway obstruction did not improve even two days after surgery. Hence, dehydration therapy and tracheotomy were performed in the supine position. Three days after surgery, the patient was in a hazy consciousness state. CT showed an ischemic infarction at the bilateral internal carotid arteries and posterior cerebral artery (Figure 1C), which was not present on day 1 (Figure 1B). Magnetic resonance angiography (MRA) showed stenosis at the carotid siphon and distal basilar artery, which was segmental (Figure 1D). Hyperdynamic hemodilutional therapy and nimodipine were started. Four days after surgery, the patient was in coma state. His body temperature was $39^{\circ} \mathrm{C}$, and hence physical cooling was performed. Penicillin was replaced with vancomycin and ceftazidime. The patient received $50 \mathrm{~mL}$ of nimodipine q 12 h, hydroxyethyl starch 130/0.4, and $4.5 \mathrm{~g}$ of sodium chloride ( $500 \mathrm{~mL} /$ day), and the amount of fluid was increased to 3,000-3,500 mL/day. Five days after surgery, the level of consciousness of the patient was not improved.
There was no clear liquid exudate at the incision. The dressing was clean and dry. Six days after surgery, the patient was unconscious. CT revealed that the infarction was located in the frontal, parietal, occipital, and temporal lobes (Figure $1 \mathrm{E})$. Seven days after surgery, the level of consciousness was not improved. The infarction size increased. Dehydration therapy was carried out, without success. Decompressive craniectomy was successfully performed in the frontal and temporal parts (Figure 1F). Head dressing was clean and dry. The bone window was correctly replaced, the trachea incision was unobstructed, and the patient had small amounts of sputum. However, the patient was in a persistent vegetative state, with normal vital signs.

The Karnofsky performance status score before surgery was 90 . It was 30 on day 1 after surgery; 20 on day 2 after surgery; 10 on day 7 after surgery; and 40 on the last follow-up (ie, five years after surgery).

\section{Discussion}

The most common predisposing factor involved in vasospasm occurrence after intracranial tumor resection is subarachnoid hemorrhage (SAH). ${ }^{5}$ Blood accumulation in the basal cisterns is recognized as an essential factor to initiate the vasospasm. ${ }^{5,6}$ The trigeminal system regulates blood flow and metabolism of the brain, and it seems to be an important element involved in cerebral vasospasm. Stimulation of trigeminal nerve endings induces vasodilation and upregulation in cerebral blood flow. ${ }^{7}$ Direct mechanical trauma, vasoactive substances, tumor location, and tumor compression are considered to be the likely causes of vasospasm. ${ }^{8}$

In the present case, the tumor was completely resected without active bleeding, in contrast to what is sometimes seen in vasospasms after tumor resection. ${ }^{5,6}$ In addition, the cerebral vasospasm occurred three days after surgery, which is not consistent with the occurrence of SAH-associated vasospasm. On the other hand, direct mechanical trauma to the arterial wall (especially microvessels) during tumor resection was inevitable and could induce local arteries vasoconstriction, which would result in an intrinsic myogenic response, upregulated norepinephrine levels, or increased cerebrovascular reactivity to norepinephrine and serotonin. ${ }^{3}$ Contractility and elasticity of arterioles are better in young people, which might make them more sensitive to direct mechanical trauma. Indeed, older patients ( $\geq 68$ years of age) with aneurysmal SAH have a lower incidence of symptomatic vasospasm. ${ }^{9}$ The occurrence of vasospasm in the present case might be secondary to the age-related response to mechanical stimulation or vasoactive substances. 


\section{A}
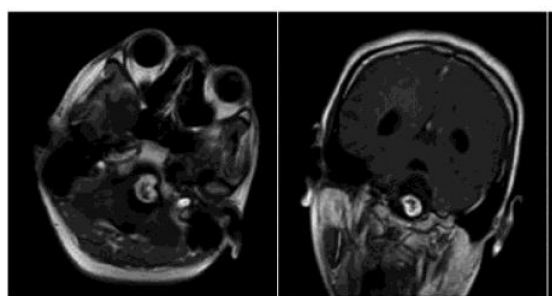

B

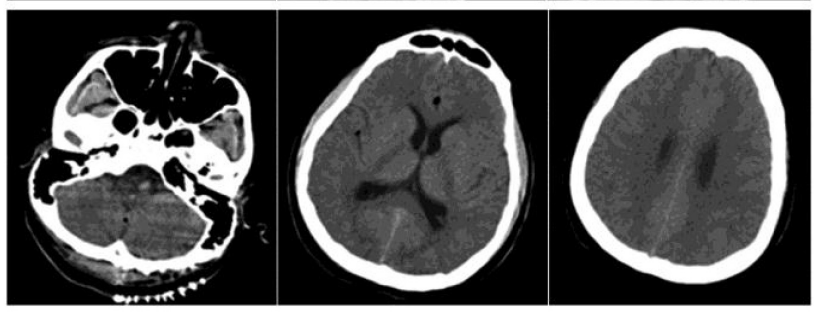

C

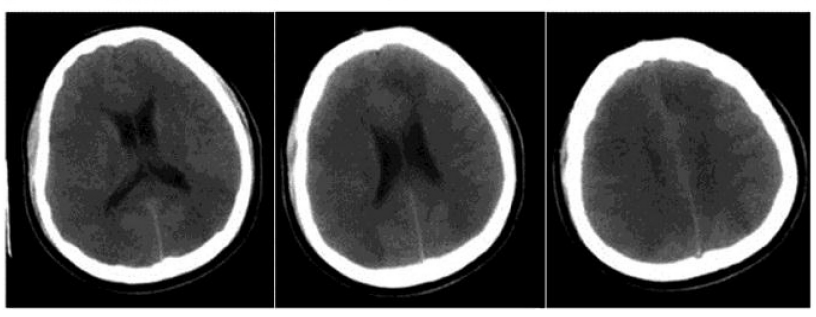

D

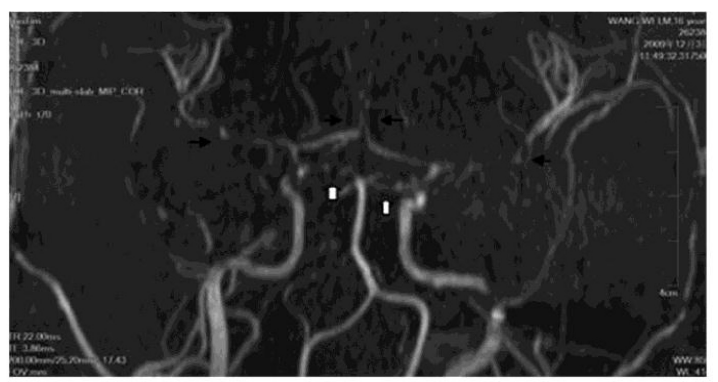

E

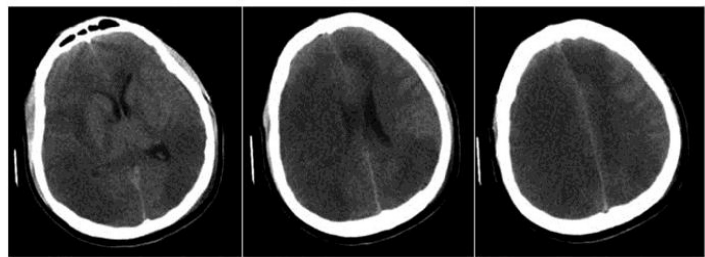

$\mathbf{F}$

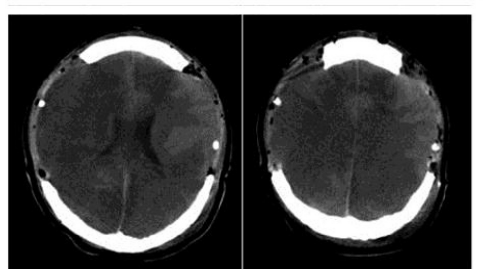

Figure I Preoperative and postoperative findings by MRI and CT.

Notes: (A) Preoperative MRI scan showing the tumor located in the left part of the ventral medulla oblongata. (B) Postoperative day I CT scan showing no apparent ischemic infarct area. (C) Postoperative day 3 CT scan showing an ischemic infarction at the bilateral internal carotid arteries and the posterior cerebral artery. (D) Postoperative day 3 MRA showing stenosis in the siphon segment of the bilateral internal carotid arteries and distal segment of basilar artery, and the slender distal blood vessel. Cerebral artery showed a slender, segmental stricture. (E) Postoperative CT scan showing the progressively enlarged ischemic infarction area. (F) Postoperative CT scan showing decompressive craniectomy.

Abbreviations: CT, computed tomography; MRA, magnetic resonance angiography; MRI, magnetic resonance imaging.

As far as we know, this is the first report of cerebral vasospasm after the resection of a schwannoma located in the ventral medulla, which is rare. Studies suggest that tumor location might contribute to the development of vasospasm, ${ }^{1,8,10-12}$ especially if the tumor is in the vicinity of the trigeminal nerve, A2 nucleus, and/or median eminence. A review suggests that pituitary tumors are most likely to induce the cerebral vasospasm. ${ }^{3}$ Indeed, MRI revealed that

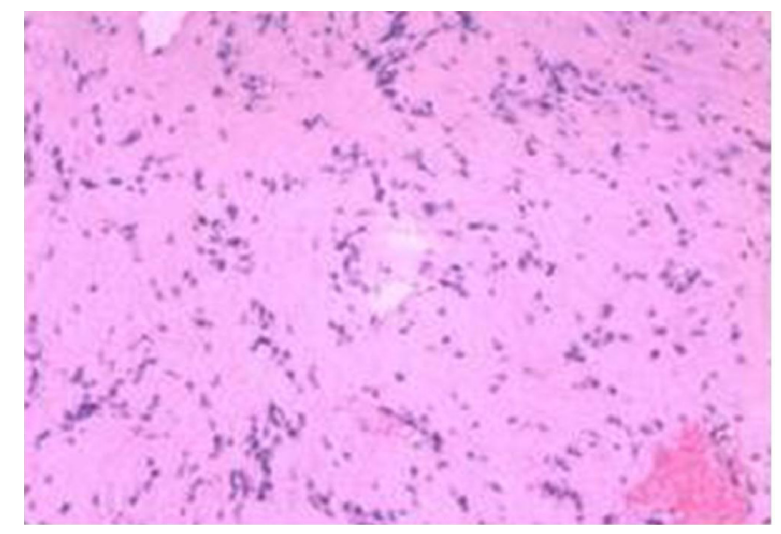

Figure 2 Photomicrograph of the tumor specimen.

Note: Pathological examination was consistent with a schwannoma. the lesion was in the left part of the ventral medulla oblongata, completely compressing the medulla oblongata, with edema. It is likely that tumor removal stimulated the A2 nucleus or the release of vasoactive substances from the tumor bed, affecting the postganglionic trigeminal system, A2 nucleus, and the median eminence.

Aggressive treatment methods were used in the present case, preventing death, but not important morbidity. Therefore, the appropriateness of these treatments might be questionable, and prophylactic treatments might be more appropriate. However, there is no study about the use of vasodilator and cerebral perfusion after surgery of brainstem tumors. In the present study, the successful use of vasodilator, cerebral perfusion, and surgery were limited after the onset of cerebral vasospasm.

\section{Author contributions}

JQ, LZ: substantial contributions to conception and design, acquisition of data, or analysis and interpretation of data. All authors contributed toward data analysis, drafting and revising the paper and agree to be accountable for all aspects of the work. 


\section{Disclosure}

The authors report no conflicts of interest in this work.

\section{References}

1. Aoki N, Origitano TC, al-Mefty O. Vasospasm after resection of skull base tumors. Acta Neurochir (Wien). 1995;132(1-3):53-58.

2. Lee TT, Ragheb J, Bruce JC, Altman N, Morrison G. Diffuse cerebral vasospasm with ischemia after resection of a cerebellopontine angle primitive neuroectodermal tumor in a child. Pediatr Neurosurg. 1998; 29(6):300-303.

3. Alotaibi NM, Lanzino G. Cerebral vasospasm following tumor resection. J Neurointerv Surg. 2013;5(5):413-418.

4. Takei H, Schmiege L, Buckleair L, Goodman JC, Powell SZ. Intracerebral schwannoma clinically and radiologically mimicking meningioma. Pathol Int. 2005;55(8):514-519.

5. Bejjani GK, Sekhar LN, Yost AM, Bank WO, Wright DC. Vasospasm after cranial base tumor resection: pathogenesis, diagnosis, and therapy. Surg Neurol. 1999;52(6):577-583. [discussion 583-574].

6. Prontera A, Puzzolante A, Carpeggiani P, Pavesi G. Symptomatic anterior cerebral artery vasospasm after brainstem hemangioblastoma resection. A case report. Neuroradiol J. 2014;27(2):186-190.
7. Atalay B, Bolay H, Dalkara T, Soylemezoglu F, Oge K, Ozcan OE. Transcorneal stimulation of trigeminal nerve afferents to increase cerebral blood flow in rats with cerebral vasospasm: a noninvasive method to activate the trigeminovascular reflex. $J$ Neurosurg. 2002;97(5): $1179-1183$.

8. Jacob JT, Hunt CH, Wijdicks EF, Rabinstein AA, Cloft H, Link MJ. Diffuse cerebral vasospasm after resection of a posterior fossa ependymoma. Neurocrit Care. 2011;14(1):86-90.

9. Torbey MT, Hauser TK, Bhardwaj A, et al. Effect of age on cerebral blood flow velocity and incidence of vasospasm after aneurysmal subarachnoid hemorrhage. Stroke. 2001;32(9):2005-2011.

10. Chang SD, Yap OW, Adler JR Jr. Symptomatic vasospasm after resection of a suprasellar pilocytic astrocytoma: case report and possible pathogenesis. Surg Neurol. 1999;51(5):521-526. [discussion 526-527].

11. de Almeida GM, Bianco E, Souza AS. Vasospasm after acoustic neuroma removal. Surg Neurol. 1985;23(1):38-40.

12. Barrow DL, Tindall GT. Loss of vision after transsphenoidal surgery. Neurosurgery. 1990;27(1):60-68.

\section{Publish your work in this journal}

Neuropsychiatric Disease and Treatment is an international, peerreviewed journal of clinical therapeutics and pharmacology focusing on concise rapid reporting of clinical or pre-clinical studies on a range of neuropsychiatric and neurological disorders. This journal is indexed on PubMed Central, the 'PsycINFO' database and CAS, and is the official journal of The International Neuropsychiatric Association (INA). The manuscript management system is completely online and includes a very quick and fair peer-review system, which is all easy to use. Visit http://www.dovepress.com/testimonials.php to read real quotes from published authors. 